\title{
Le résumé scientifique — texte monophonique ou polyphonique?
}

\author{
Kjersti Fløttum \\ Université de Bergen, Norvège
}

In this article, I study the scientific abstract, traditionally considered as impersonal and non-engaged, in the perspective of linguistic polyphony (Ducrot 1984, Nolke 1994). My purpose is to identify elements which manifest authorial presence in the abstract and thereby to test the hypothesis that there are traces of commitment and discussion in the scientific abstract. The results show that this type of abstract is not monophonic. Commitment and discussion are not absent.

Dans cet article, j'étudie le résumé scientifique, traditionnellement considéré comme impersonnel et non-engagé, dans la perspective de la polyphonie linguistique (Ducrot 1984, Nølke 1994). Mon objectif est de repérer des éléments manifestant la présence d'un locuteur dans le résumé scientifque et ainsi de tester l'hypothèse selon laquelle le résumé scientifique contient des marques d'engagement et de discussion. Les résultats démontrent que ce type de résumé n'est pas monophonique et que l'engagement et la discussion y sont présents.

\section{Introduction}

\subsection{Objectif}

Qui parle dans les résumés scientifiques ? Voilà la question, assez simple dans sa forme mais complexe dans ses implications, à laquelle je voudrais essayer de répondre dans cet article.

Tout d'abord, il faudra préciser que les résumés scientifiques étudiés ici sont généralement du type informatif, par opposition au résumé indicatif (pour cette distinction, voir Fløttum 1990 et Dahl 200ob). Les résumés indicatifs ne contiennent normalement que des éléments portant sur l'objectif et la méthode et/ou les matériaux de la recherche exposée dans l'article source, tandis que les résumés informatifs 
doivent contenir des éléments portant sur tous les composants de la structure de la norme IMRAD, c'est-à-dire des éléments portant également sur les résultats ainsi que sur la conclusion/discussion (pour la norme IMRAD, voir 1.2).

Retournons à la question initiale : qui parle dans les résumés scientifiques ? S’il faut en croire les consignes formulées par l'Organisation internationale de normalisation, dans ISO-214-1976 (F), personne ne parle dans ces résumés (ou " analyses ») :

Dans la présente Norme internationale, le terme analyse signifie une représentation abrégée et précise du contenu d'un document, sans interprétation ou critique et sans distinction de l'auteur de l'analyse (ISO 1976 :37).

Quoi qu'il en soit, que l'auteur ${ }^{1}$ soit précisé ou non, il y a toujours un locuteur qui est responsable des énoncés émis. En prenant ce fait comme point de départ de la présente étude, mon objectif pourra être précisé comme suit : d'abord, j'examinerai dans quelle mesure et de quelle manière le locuteur se manifeste dans le résumé scientifique - dans quelle mesure il existe un engagement de la part du locuteur ; ensuite j'étudierai l'hypothèse de la présence d'autres voix ou points de vue dans le résumé et dans quelle mesure cette présence éventuelle crée une discussion. Les résultats contribueront à répondre à la question formulée dans le titre : le résumé scientifique est-il un texte monophonique ou polyphonique?

Cet article s'appuie sur la théorie de la polyphonie linguistique (voir 2), telle qu'elle est développée par Ducrot (1984) et élaborée par Nølke $(1994,1999)$. La polyphonie linguistique étant une rencontre de plusieurs points de vue mis en scène par le locuteur, elle semble tout indiquée pour la présente étude. Cette recherche s'inscrit dans un projet nordique sur lequel travaillent sept chercheurs - trois linguistes et quatre littéraires - coopérant dans le domaine de la Polyphonie linguistique et littéraire (voir par exemple Tribune 9, 1999, et notre site internet http://www.hum.au.dk/ romansk./polyfoni/).

\subsection{Objet d'étude - hypothèse}

Tout d'abord, il faut préciser tant soit peu la problématique soulevée. A première vue, les résumés scientifiques publiés dans diverses revues semblent être impersonnels, non-engagés et absents de toute discussion. C'est le style scientifique dit objectif qui domine. Cependant, en les analysant de plus près, on y décèle certains phénomènes linguistiques signalant une forme d'engagement ainsi que des éléments de discussion. Par engagement, j'entends la présence de l'auteur telle qu'elle se manifeste a) par des pronoms personnels de première personne et $b$ ) par des éléments lexicaux valorisés. Par discussion, j'entends l'échange de points de vue manifestée c) à l'aide de références à des travaux antérieurs entrepris soit par l'auteur lui-même, soit par d'autres 
auteurs engagés dans d'autres recherches (les noms et/ou dates de parution liés à d'autres recherches) et, d'une manière moins directe, d) par des constructions syntaxiques déterminées et des connecteurs pragmatiques.

Pour vérifier mon hypothèse de travail, selon laquelle le résumé scientifique contient des marques d'engagement et de discussion, il faut s'appuyer sur une étude approfondie. Cet article ne représente que le début modeste d'une telle étude. De caractère exploratoire, ma recherche vise à repérer des phénomènes linguistiques pertinents pour appuyer l'hypothèse formulée. Le caractère et l'étendue des matériaux (voir 3 ) ne permettront pas non plus de faire une analyse quantitativement valable.

Fondamentalement, mon hypothèse s'appuie sur la conception même de l'article scientifique. Ce dernier est de nature polémique et argumentatif et mène à une discussion. Étant donné que le résumé d'un article est une représentation plus ou moins fidèle du texte source, il est raisonnable de supposer que cette polémique se manifeste d'une manière ou d'une autre dans le résumé du texte.

Bien que le style neutre et non-engagé prévaut dans les travaux scientifiques, peut-être plus pour certaines disciplines que pour d'autres, la discussion y est bien présente. Il existe bien entendu des différences notables entre diverses disciplines (voir Bazerman 1988, Breivega 2000 et à paraître). Cependant, si les éléments contribuant à l'argumentation et à la discussion sont inégalement répartis dans les articles de disciplines différentes, ils sont « obligatoirement » présents dans la partiediscussion de tout article. Je renvoie ici à la norme IMRAD, internationalement reconnue, quoique se prétant mieux pour les sciences naturelles. Cette norme, imposée aux auteurs d'articles scientifiques, établit les composantes suivantes : introduction, méthode/matériaux, résultats et discussion. Pour la présente étude, il sera important de voir dans quelle mesure la discussion est représentée dans le résumé de l'article, que cette discussion soit située à un endroit précis ou à plusieurs endroits dans l'article source.

Par ailleurs, certains facteurs justifient le fait de porter attention au résumé scientifique en général et à ses aspects énonciatifs en particulier. Tout d'abord, l'intérêt pour le résumé scientifique (ou abstract dans la tradition anglo-américaine) ne cesse de s'accroître depuis ces dernières années. Pour un aperçu des recherches déjà effectuées, je renvoie à Dahl (200oa et 200ob) et Lindeberg (à paraître), qui traitent également de l'importance et de l'utilité incontestables de ces résumés - importance due à la croissance énorme d'articles scientifiques publiés dans des revues internationales diffusées dans le monde entier. De plus, le milieu scientifique croit à l'importance des résumés scientifiques compte tenu du nombre d'articles scientifiques publiés de nos jours auxquels on ne peut accéder que par leur résumé (voir aussi Fløttum 1990 :12, où est soulignée l'utilité de l'enseignement portant sur le résumé de texte en général). 
Bien que l'importance des résumés scientifiques soit incontestable, la manière de les rédiger et de les formuler sont absentes dans la plupart des revues scientifiques. Certains normes, dont celle de ISO-214 (1976), proposent de structurer le contenu en objectif, méthode, résultats et conclusions. Il existe également des paradigmes différents élaborés par des chercheurs travaillant sur ces résumés (voir Lindeberg à paraître et Dahl 200ob). Cependant, on trouve peu de directives sur les aspects énonciatifs, voire sur le style des résumés. Dans quelle mesure ces résumés doivent-ils être caractérisés par un style neutre et objectif ? Les éléments d'engagement subjectif sontils permis ? Que signifie la remarque suivante formulée dans ISO-214 (1976:40) : « Il faut employer la troisième personne à moins que l'usage de la première personne n'evite les constructions gênantes de phrases et [n'] aboutisse à une plus grande clarté de compréhension "? Bien qu'il existe très peu de directives, il semble y avoir un consensus en faveur du non-engagement des abstracts ou résumés scientifiques. On peut se demander si c'est une conception démodée de la recherche dite objective qui règne toujours.

Il est également à noter que dans la grande majorité des études entreprises sur les résumés, l'intérêt porte sur les composants de contenu (pour des études intéressantes dans ce domaine, voir entre autres Dahl 200ob, Lindeberg à paraître, Tibbo 1992, Liddy 1991), et non pas sur les aspects énonciatifs, le style ou la tonalité, et ce, malgré le conseil suivant exprimé dans ISO-214 : " retenir l'information de base el'esprit du texte original $»(1976: 40$; c'est moi qui souligne).

Dans ce contexte, il est intéressant de rappeler la conception, bien fondée, que les articles scientifiques, dans la plupart des cas, expriment l'intention de convaincre le lecteur de quelque chose - une intention qui, normalement, s'appuie sur une argumentation dans le texte. Cette argumentation peut être plus ou moins manifeste (voir Breivega 2000, Swales 1990), mais elle est bien là d'une manière ou d'une autre. Voilà pourquoi il est intéressant d'étudier dans quelle mesure cette argumentation est conservée dans le résumé de l'article. Dans les études que j’ai entreprises sur le résumé scolaire (Fløttum 1990), les résultats ont indiqué clairement que ce type de résumé conserve l'argumentation et ainsi le fondement de l'intention exprimée dans le texte source. Il peut sembler que les résumés scientifiques ne soient pas si fidèles au texte source que les résumés scolaires ; il faut pourtant se rappeler que la contrainte de la longueur peut jouer un rôle important dans ce contexte. Le résumé scientifique est soumis à des contraintes de longueur beaucoup plus strictes (souvent de 5 à 10 lignes) que le résumé scolaire. De telles contraintes orientent bien entendu le contenu du résumé. 
On peut également mentionner que certains chercheurs (dont Berkenkotter \& Huckin 1995) signalent une différence importante entre les habitudes de lecture des chercheurs et la structure des articles scientifiques. En constatant que les chercheurs ont l'habitude de repérer d'abord l'information nouvelle et intéressante, habitude correspondant d'une manière très semblable à l'habitude des lecteurs de journaux (" top-down "), ils affirment que la correspondance entre le schéma de lecture spécialisée et le schéma écrit traditionnel des articles scientifiques, où les résultats et la discussion sont situés à la fin, est loin d'être optimale :

After all, the reader has to search for information, rather than just proceeding top-down. The only text features that facilitate a top-down approach are the Title and Abstract, which appear first and are read first [...]. Newspaper articles, by contrast are noticeably reader based [...], they pick out the most surprising information and put it up front (Berkenkotter \& Huckin 1995 :32).

Une question intéressante dans ce contexte, mais qui ne sera pas étudiée ici, pourrait se formuler ainsi : le résumé peut-il être considéré comme le « chapeau " de l'article de presse ? (Voir aussi l'étude de Frandsen 1992 portant sur la structure des textes journalistiques.)

\section{Cadre théorique}

Comme je l'ai déjà indiqué, cette recherche s'insère dans une perspective énonciative des textes (voir aussi Laurendeau 1990). J'adopte, depuis un certain temps, un point de vue qui donne plus d'importance aux faits énonciatifs des textes, plus particulièrement dans le cadre de la typologisation de texte (Fløttum 1999a, b, à paraître a, b). Dans cette perspective énonciative, la théorie de la polyphonie linguistique pourra, à mon avis, constituer une importante contribution. Si l'on met l'accent sur le module énonciatif d'un modèle typologique tel que celui qui est proposé par Adam (1992), et plus précisément sur la notion de polyphonie, le résultat pourra être une distinction nouvelle à l'intérieur de chaque type de texte, comme par exemple pour le type descriptif : descriptif d'énonciation polyphonique (exemple de genre :publicité) versus descriptif d'énonciation non-polyphonique (exemple de genre : manuel de géographie), et ainsi de suite pour le type narratif, le type argumentatif et le type explicatif (voir Fløttum à paraître b). À mon avis, la distinction polyphonique/non-polyphonique est tout indiquée pour les grandes masses textuelles, pour les genres littéraires et non-littéraires. Tout texte peut se situer selon l'axe polyphonique/non-polyphonique, même s'il se classe difficilement comme argumentatif, descriptif ou autre. 
Considérons maintenant quelques principes de la version linguistique de la théorie de la polyphonie, développée par Ducrot (1984) et élaborée par Nølke (1994 et 1999). Un des objectifs les plus importants de cette théorie est de contester le postulat affirmant l'unicité du sujet parlant (voir Ducrot 1984 171). On se propose de montrer comment la phrase signale la superposition de plusieurs points de vue (c'est Nølke qui adopte le terme de point de vue pour le phénomène qui est aussi désigné par les termes de voix (la tradition de Bakhtine) ou d'énonciateur (voir Nølke 1994 :148-149)). Pour l'interprétation d'un énoncé concret, il s'agit donc de repérer les différents points de vue qui se manifestent et, à un stade ultérieur, d'identifier les êtres discursifs qui constituent l'origine de ces points de vue. Il peut s'agir d'un point de vue du locuteur, être discursif responsable de l'énoncé, ou d'un point de vue de l'allocutaire, être discursif auquel est destiné l'énoncé, ou encore d'autres points de vue ayant comme origine différents êtres discursifs (l'opinion générale, des groupes socio-économiques déterminés, etc.). C'est le locuteur qui est responsable de l'énoncé et qui peut présenter plusieurs points de vue dans un seul et même énoncé. Ces points de vue peuvent à leur tour entretenir des relations différentes avec le locuteur. Pour l'interprétation d'un énoncé isolé aussi bien que pour un texte consistant en plusieurs énoncés, il est particulièrement intéressant de déterminer ces relations et les êtres discursifs qui en sont l'origine. $\AA$ mon avis, c'est là un facteur tout à fait décisif pour la cohérence du texte (Fløttum 1997).

Dans une introduction à la théorie de la polyphonie linguistique, il faut également mentionner la distinction que fait Nølke (et Ducrot) entre, d'une part, le locuteur-en-tant-que-tel, l, qui est responsable de l'énoncé et qui n'existe que dans l'événement énonciatif et, d'autre part, le locuteur-en-tant-qu'individu, L, qui a une existence indépendante de l'événement énonciatif en même temps qu'il a la qualité d'être locuteur-en-tant-que-tel d'un énoncé (voir Nølke 1994 :152). Le locuteur-en-tantque-tel de chaque énoncé peut être considéré comme différentes images du locuteuren-tant-qu'individu. Ce dernier constitue donc encore une unité fondamentale pour la cohérence textuelle. Dans une étude du résumé scientifique plus générale que celle entreprise ici, cette division du locuteur pourra se révéler essentielle.

Pour illustrer ces différents termes et notions, je présenterai l'analyse polyphonique de la négation, reprenant l'exemple canonique de Ducrot :

(1) Ce mur n'est pas blanc.

Sans entrer dans la discussion des différentes fonctions de la négation syntaxique ne...pas, je précise seulement qu'il s'agit ici, bien entendu, de la négation polémique (voir Fløttum 1998). Dans cette phrase (1), deux points de vue (pdv) sont présentés : 
pdv1 : Ce mur est blanc.

pdv 2 : pdv1 est injustifié

Le locuteur est responsable du pdv 2 et se dissocie du pdv 1. Il s'agit donc d'une relation de dissociation et réfutation ou de non-responsabilité entre le locuteur et le pdv 1 et une relation d'association ou de responsabilité entre le locuteur et le pdv 2.

Une autre relation qui peut se manifester entre les pdv et le locuteur est la relation d'accord, comme par exemple dans une construction concessive du type Certes..., mais..., où certes signale l'accord et mais l'association au point de vue qui suit (voir Nølke 1994 :150-151).

Dans mes études antérieures, $j$ 'affirme qu'une des raisons de l'utilité de la notion de polyphonie dans les analyses linguistiques est sa capacité d'indiquer des relations hiérarchiques entre divers points de vue (c'est là, bien entendu, un aspect qui n'est pas dérivé de la conception de polyphonie développée par Bakhtine dans ses études sur Dostoïewski, qui parle de points de vue ou " voix " comme étant de valeur égale, " indépendantes ", " pleinement valables "(Bakhtin970 :10-11)). Cette capacité de signaler des relations hiérarchiques s'explique par le rôle double du locuteur : il constitue lui-même un point de vue et manipule les autres points de vue présents.

Cette perspective a été importante dans mes études du résumé de texte liées à ma thèse de doctorat (Fløttum 1990). En fait, pour résumer, il faut repérer l'organisation d'informations hiérarchique du texte original et sélectionner l'information essentielle. Par rapport à des analyses de macro- et superstructures, qui sont tout à fait importantes dans cette perspective, l'analyse polyphonique introduit une nouvelle dimension. Il semble raisonnable de supposer que pour comprendre et résumer un texte, il faut repérer les divers points de vue exprimés. Dans un article (Fløttum 1992), je propose l'hypothèse suivante : les points de vue auxquels le locuteur s'associe sont les plus importants (ou les plus forts) et donc ceux qui doivent être conservés dans le résumé ; les points de vue desquels le locuteur se dissocie sont les moins importants (ou les plus faibles) et ceux qui doivent être effacés. La relation d'accord occupe une position intermédiaire (quand le locuteur accepte un point de vue sans en prendre la responsabilité). Mon hypothèse est plus ou moins confirmée par une analyse que j'ai entreprise sur un texte authentique, comparé avec son résumé. Cette analyse a donné des résultats intéressants, qui justifient l'application de la théorie de la polyphonie dans l'étude du résumé de texte.

Dans la présente étude, la problématique se présente différemment. Le résumé scolaire et le résumé scientifique sont des textes construits de manière différente. Pour l'étudiant rédigeant un résumé dans un contexte scolaire, il s'agit de faire une version aussi fidèle que possible d'un texte écrit par un autre. Pour le chercheur, il s'agit de 
faire une version rendant l'essentiel du texte original écrit par lui-même (sauf dans les cas où ce sont des analystes professionnels qui rédigent le résumé). Dans la présente étude, il ne s'agira pas d'une comparaison entre texte original et résumé (comme dans Fløttum 1990 et 1992) ; les questions posées ici sont liées uniquement aux aspects énonciatifs du résumé.

\section{Corpus d'analyse}

Le corpus d'analyse est constitué d'une centaine de résumés français d'articles scientifiques, tirés de diverses revues de disciplines différentes parues entre 1984 et 1999 (voir liste des revues ci-dessous). Il s'agit de revues de langue française seulement ou de revues qui acceptent des articles en plusieurs langues, dont le français. Dans ces revues, il peut $y$ avoir des résumés écrits non seulement en français, mais dans d'autres langues comme l'anglais (assez souvent), l'espagnol et l'allemand. Pour des raisons pratiques aussi bien que méthodologiques, je ne considère que les résumés écrits en français ici. Toutefois, il serait intéressant de comparer ultérieurement deux ou trois résumés d'un même article rédigés dans des langues différentes.

Je tiens à souligner que ces matériaux sont choisis en fonction de la présente étude qui est de caractère exploratoire. Bien qu'il soit tentant de comparer les résumés de différentes disciplines (pour une telle étude, voir Tibbo 1992), ni la nature de notre étude ni le nombre de résumés ne permettent une telle analyse.

Voici la liste des revues ( $\mathrm{y}$ compris, entre parenthèses, les sigles utilisés dans le texte ci-dessous) dont sont tirés les résumés étudiés :

Annales de médicine interne, 1984, 135 (1), Paris : Masson(AMédI)

L'année biologique, 1994, 33 (3-4), Paris : Masson (ABio)

Comptes rendus de l'Académie des sciences - Mathématique, 1999, 329 (12), Paris :

Elsevier (CRMath)

Comptes rendus de l'Académie des sciences - Chimie, 1999, 2 (11-13), Paris : Elsevier (CRChim)

Annales d'économie et de statistique, 1999, 54, Paris (AEStat)

Canadian Journal of Economics - Revue canadienne d'économie, 1999, $32(1,3,4)$

Toronto : University of Toronto Press (CJEcon)

Annales de géographie, 1984, 515, Paris (AGéo)

L'espace géographique, 1999, 28 (4), (EGéo)

Population, 1999, 54 (1), Paris (Pop)

Revue française de sociologie, 1999, 40 (4), Paris : Ophrys (RFSoc)

Recherches amérindiennes au Québec, 1999, 29 (2), Québec (RAQuéb)

Revue française de science politique, 1999, 49 (6), Paris : Presses de ScPo., (RFScpo) 
Revue internationale d'histoire militaire, 1997, 76 (RIHMil)

Revue des études anciennes, 1999, 101 (3-4), Bordeaux (REAnc)

Revue archéologique, 1999, 2, Paris, PUF, (RArch)

Revue historique 199, 301 (611), Paris, (RHist)

Pratiques, 1997, $94+$ 1999, 103-104, Metz, (Prat)

Travaux de linguistique, 1994, $29+1999,38$, Bruxelles (TLing)

\section{Méthode d'analyse}

Le cadre théorique de cette étude est limité à la linguistique énonciative en général et à la polyphonie linguistique en particulier. Pour une étude plus générale du résumé scientifique, il faudrait prendre en considération d'autres perspectives ; il serait intéressant d'étudier l'interaction de perspectives syntaxiques, sémantiques, pragmatiques et textuelles (pour une approche modulaire de ce genre, voir Fløttum 1996). Dans la présente analyse, il s'agira de repérer, dans des revues de différentes disciplines, des marques polyphoniques dans un sens large, ainsi que des marques d'engagement et de discussion.

Pour les marques d'engagement, j'examinerai la présence de l'auteur telle qu'elle se manifeste à travers

a) les pronoms personnels de première personne, et

b) des éléments lexicaux valorisés.

Exemple de a :

(2) Nous trouvons peu de support pour cette hypothèse ${ }^{2}[\ldots]$ (CJEcon 1999, 32 (4), 843).

Pour qu'il y ait polyphonie, il faut qu'il y ait au moins un locuteur qui puisse organiser les différents points de vue. Dans une perspective polyphonique, l'intérêt des exemples tels que (2) est l'explicitation d'un être discursif (voir Norén 1999, p. 9899). Dans ce cas précis, c'est l'être discursif principal, le locuteur, qui a laissé une marque par le pronom nous.

Un autre aspect intéressant est lié à l'identification du référent. Sans développer cette discussion ici, je pourrais signaler que, dans ce cas précis, l'article en question est écrit par trois chercheurs. Vassileva (1998) a entrepris des études intéressantes concernant l'emploi et la référence des pronoms personnels de première personne dans le discours académique.

Exemple de b : 
(3) La démonstration utilisée donne un cadre clair à [...] (AEStat 1999, 54, 1).

Les éléments lexicaux valorisés ne représentent pas une rencontre de différents points de vue, mais constituent pourtant des marques bien explicites du point de vue ou plutôt de l'attitude du locuteur, comme l'adjectif clair dans l'exemple (3).

Les marques de discussion, qui expriment des échanges de points de vue, se manifestent à l'aide de :

c) références soit à l'auteur lui-même, dans l'article en question ou dans des articles antérieurs, soit à d'autres auteurs engagés dans d'autres recherches, c'est-à-dire les noms et/ou dates de parution liés à ces recherches, et

d) des constructions syntaxiques déterminées et des connecteurs pragmatiques signalant des constructions polyphoniques.

Exemple de c :

(4) Ouah et Rauch (1990) suggèrent que [...] (CJEcon 1999, 32 (4), 1009).

Dans ce cas précis, Rauch est en effet un des auteurs de l'article.

Exemple de d :

(5) L'auteur examine un nouveau marché d'exportation dans lequel des entreprises peuvent investir dans la qualité mais peuvent aussi ne pas réussir à l'obtenir (CJEcon 1999, 32 (1), 39).

On peut ici noter le connecteur mais, signalant une opposition ainsi que la négation syntaxique ne pas, marquant une réfutation d'un point de vue.

Dans le cas c, on peut parler de polyphonie explicite, et dans le cas d, de polyphonie implicite, où les sources des points de vue présentés ne sont pas explicitées.

\section{Résultats}

Les analyses exploratoires entreprises ont montré que les catégories proposées pour repérer des marques d'engagement et de discussion sont suffisamment bien fondées et peuvent servir de base à des recherches ultérieures plus approfondies. Passons maintenant aux résultats concrets des analyses. Nous allons d'abord considérer la catégorie de l'engagement.

Pour ce qui est de la présence de l'auteur ou des auteurs, elle peut se manifester par le pronom nous: 
(6) Nous étudions le comportement asymptotique [...] Nous traitons ici les cas des processus gaussiens [...] nous obtenons la convergence vers une courbe limite convexe [...] (CRMath 1999, 329 (12), 1087).

Dans cet exemple, il s'agit d'un article et d'un résumé écrit par deux auteurs ; dans le suivant, le pronom nous réferre à un seul auteur :

(7) Nous donnons les conditions d'existence d'un équilibre stationnaire [...] (AEStat 1999, 54, 1).

Dans une étude portant sur l'engagement de l'auteur, il ne suffit pas, bien entendu, d'observer la seule présence du pronom nous. Il est généralement admis que ce pronom peut avoir plusieurs valeurs, dont le pluriel de majesté et le pluriel de modestie (voir Vassileva 1998 : 173 et Loffler-Laurian $1980: 156$ ). Pour la présente étude, je me contente d'observer la présence de ce pronom dans les résumés scientifiques.

Pour ce qui est du pronom je, je n'ai pas pu en repérer un seul exemple dans les résumés étudiés. C'est là une observation qui ne doit pas nous étonner. Dans la tradition du discours académique français, la première personne du singulier est évitée (voir Loffler-Laurian 1980). Sans essayer d'expliquer ce fait, je suis tentée de rappeler que dans la tradition académique anglo-américaine, l'emploi du pronom I est tout à fait de mise.

À côté de nous, le pronom on est attesté dans de nombreux résumés, où il est également difficile de déterminer sa référence précise :

(8) On a examiné plus particulièrement les conséquences [...] (CRChim 1999, 2 $(11-13), 669)$.

Cependant, dans l'exemple suivant, il semble clair que on renvoie à la communauté discursive en question, dans son ensemble, le locuteur inclus :

(9) Dans le discours actuel sur la presse écrite, on constate une tendance à catégoriser comme " récit " un grand nombre de genres journalistiques [...] (Prat, $1997,94,121)$.

Quelle que soit la référence précise des pronoms nous et on, le locuteur marque nettement sa présence en les utilisant.

Procédons maintenant à la présence de l'auteur telle qu'elle se manifeste à travers des éléments lexicaux valorisés. Dans ce contexte, il peut être intéressant de faire une distinction entre éléments positifs et éléments négatifs. Dans l'exemple (10), le subs- 
tantif inadéquation a une valeur négative, qui pourra orienter l'argumentation dont elle fait partie dans un sens précis (pour la « Théorie de l'argumentation dans la langue ", voir par exemple Anscombre \& Ducrot1983) :

(10) L'inadéquation de cette théorie en rapport avec les données récentes [...], stimula l'émergence de nouvelles théories [...] (ABio 1994, 33 (4), 221).

Dans l'exemple suivant, le substantif utilité peut avoir une valeur positive,

(11) Voilà qui souligne l'utilité d'analyses transversales des taux de change pour plusieurs pays (CJEcon 1999, 32 (1), 118).

ainsi que l'adverbe soigneusement dans (12) :

(12) Il distingue soigneusement les genres de la presse écrite des autres unités rédactionnelles [...] (Prat, 1997, 94, 121).

Si un élément lexical valorisé n'est pas polyphonique en soi, il manifeste toutefois un engagement du locuteur, engagement qui joue un rôle important dans l'impression que le résumé donne au lecteur.

Procédons maintenant aux résultats concernant la catégorie de discussion. L'analyse entreprise a démontré que des échanges de points de vue se manifestent de manières différentes :

- Par une référence, à l'aide de l'expression comme on sait, à un point de vue traduisant une connaissance générale :

(13) La mortalité par suicide, comme on sait, est particulièrement élevée en France [...] (Pop1999, 54 (1), 73).

- Par une référence à un travail antérieur fait par l'auteur lui-même :

(14) Dans un précédent article, Bernard Zarca avait montré que [...]. B. Zarca observait aussi $[. .$.$] . Une enquête plus récente lui permet de reprendre et d'ap-$ profondir ses analyses : l'héritage demeure, mais se fait un peu plus égalitaire (Pop1999, 54 (1), 37).

- Par une référence précise à des travaux entrepris par d'autres chercheurs :

(15) Dans ce mémoire, les prédictions proposées dans les analyses de jeux à deux étapes de Copeland (1990) et Copeland, Tower et Webb (1989) sont analysées empiriquement $[\ldots]$. La première ronde des résultats obtenus $[\ldots]$ indique certaines directions pour le travail théorique qui reste à faire (CJEcon 1999, 32 (4), 1028). 
- Par une référence à des chercheurs précis mais sans indication de publication et date :

(16) Dans cet article nous traitons du pouvoir explicatif des rôles discursifs. Nous comparons deux classifications, celles de Ducrot et de Sells. Il s'avère que leurs rôles sont apparentés aux " strates " de la structure de l'énoncé proposée par Dik et Hengeveld. Ce cadre théorique est utilisé pour expliquer [...] (Tling, 1994, 29, 1).

- Par une référence à des travaux antérieurs sans indication de chercheur(s) et date de parution :

(17) L'une des thèses actuellement les plus discutées dans le champ de la science politique et de la sociologie politique est celle de la recomposition des États modernes. Parmi les processus [...] figurerait le déplacement dans la localisation du politique. Le niveau national [...] perdrait de sa centralité [...]. Cet article se fixe comme objectif de tester l'hypothèse de l'autonomisation progressive des villes européenes [...] (RFScpo 1999, 49 (6), 853).

- Par une référence tout à fait non spécifiée :

(18) Des recherches récentes apportent des données nouvelles [...] (ABio 1994, 33 $(3), 154)$.

Toutes ces références, quoique plus ou moins précises, indiquent nettement l'échange de points de vue qui a lieu dans le monde scientifique. Cependant, il y a lieu de discuter dans quelle mesure elles constituent les parties d'une véritable discussion. Les références peuvent être assez neutres, comme au début de l'exemple (15) : « Dans ce mémoire, les prédictions proposées dans les analyses de jeux à deux étapes de Copeland (1990) et Copeland, Tower, et Webb (1989) sont analysées empiriquement " ; mais elles constituent un point de départ net pour une discussion entre l'auteur et les travaux de recherche auxquels renvoient les noms cités: « [...]. La première ronde des résultats obtenus $[\ldots]$ indique certaines directions pour le travail théorique qui reste à faire. »

Si la discussion se manifeste d'une façon explicite par ces références à d'autres chercheurs, elle se manifeste d'une façon nettement moins directe à l'aide de certaines constructions syntaxiques et de certains connecteurs pragmatiques. Il s'agit de phénomènes linguistiques qui signalent la présence, et par là l'échange, de plusieurs points de vue - il y a polyphonie. Une expression évidente dans ce contexte est celle de la négation syntaxique ne pas et non: 
(19) Ce facteur, présent dans l'hémolymphe, n'est pas spécifique du sexe ; il est de nature peptidique [...]. L'étude de la capacité post-reproductive de l'appareil génital des femelles montre que la limitation de la fécondité serait due à la mort précoce $[\ldots]$ et non à une dégénérescence de l'ovaire et du tractus génital (ABio 1994, 33 (3), 154).

À l'aide de la négation, le locuteur réfute le point de vue positif sous-jacent. Dans l'exemple suivant, à l'aide du connecteur mais, le locuteur indique qu'il accepte le point de vue qui précède ; cependant, c'est le point de vue qui suit auquel il s'associe. C'est, selon le locuteur, le point de vue le plus important :

(20) Tous les auteurs anciens [...] ont évoqué les causes du brigandage [...]. En revanche, la recherche de justice sociale est absente. Les Anciens connaissaient donc les causes du brigandage, mais ils ne les ont jamais étudiées en tant que telles et n'ont pas cherché, de façon générale, à les faire disparaître [...]. La seule prévention qu'ils connaissent était celle que procure la sévérité des peines édictées par la loi. Cela n'a pas toujours été suffisant (REAnc 1999, 101 $(3-4), 393)$.

Remarquons également, en plus de mais, les expressions en revanche, donc, et les deux négations, dans ce résumé nettement polémique.

Dans l'exemple suivant, la référence à des travaux antérieurs est introduite dans l'échange de points de vue même, manifesté par non pas et il semble plutôt que:

(21) L'auteur montre que ces différences sont engendrées par la paramétrisation de la demande et des coûts, et non pas par l'endogénéité de la qualité. Certains travaux antérieurs suggéraient que des subventions pourraient élever le niveau de bien-être. Il semble plutôt que les subventions réduisent le niveau de bien-être alors qu'une taxe peut élever le niveau de bien-être (CJEcon 1999, 32 (1), 39).

La discussion est particulièrement nette dans les cas où mais et ne pas se trouvent dans la même phrase :

(22) Au cours des dernières décennies, les spécialistes du commerce international ont construit des modèles [...] mais n'ont pas beaucoup analysé les facteurs qui expliquent la grande variation de ces élasticités de substitution d'un secteur à l'autre (CJEcon 1999, 32 (1), 1). 
Dans bien des cas, une partie polémique commence par une concession, telle que celle de l'exemple ci-dessous introduite par même si, ici suivie par une référence à des travaux antérieurs, le connecteur mais, la négation ne pas et l'expression il se peut donc $[. .$.$] .$

(23) Même si le GATT prohibe les barrières tarifaires discriminatoires à l'importation, il ouvre aussi des portes pour circonvenir cette prohibition. Les travaux antérieurs ont utilisé des modèles statistiques [...]. Mais dans un modèle dynamique, $[\ldots]$, cela n'est pas nécessairement vrai. [...]. Il se peut donc qu'il soit raisonnable $[\ldots]$ (CJEcon 1999, 32 (4), 1057).

On pourrait parler de plusieurs échanges de points de vue qui se superposent ici une accumulation de polyphonie.

Dans l'ensemble, la polyphonie se manifeste, dans ces résumés, par un nombre important de phénomènes linguistiques : la négationne pas et le connecteur mais qui sont particulièrement fréquents (voir 6. Conclusion) de même que les constructions dites impersonnelles telles que il semble, les verbes au conditionnel et une série d'expressions adverbiales et de connecteurs tels que cependant, pourtant, toutefois, néanmoins, donc, en effet, si, même si, par contre, en revanche.

Il est également à noter que les constructions à la troisième personne, souvent réalisées par un sujet tel que l'auteur (23) ou l'article ((24) où l'article est personnifié, ou encore cette étude (25)), ainsi que les constructions dites neutres et impersonnelles, comme le passif sans agent (26) ou le type il semble que (27), sont assez fréquentes :

(24) L'auteur compare [...] (CJEcon 1999, 32 (1), 152).

(25) Cet article présente un modèle [...] (AEStat 1999, 54, 1).

(26) Cette étude défend la thèse que le mode [...] est visible en syntaxe et propose dès lors un ensemble de représentations syntaxiques spécifiques pour chaque mode ; ces représentations sont définies dans le cadre de la grammaire générative $[\ldots]$ (Tling, 1999, 38, 2).

(27) Le texte choisi [...] est examiné en partant d'une première lecture [...]. L'approche énonciative permet ensuite d'en dégager la structure compositionnelle, pour finir par une analyse fine des reformulations [...] (Prat, 1997, 94, 122). 
(28) Il semble que les effets dépendent fondamentalement des préférences des syndicats ouvriers $[\ldots]$ (CJEcon 1999, 32 (1), 139).

Pour ce dernier exemple (et pour d'autres du même genre), il y a pourtant lieu de considérer de plus près la construction il semble que, qui n'est peut-être pas si impersonnelle qu'on a l'habitude de le penser. Il s'agit d'un choix précis du locuteur où il exprime une attitude modifiant les conditions de vérité du contenu sémantique exprimé dans la proposition complétive.

\section{Conclusion}

Les résultats obtenus démontrent que le cadre énonciatif et polyphonique constitue un choix fructueux pour l'étude entreprise ici, à savoir la recherche des marques d'engagement et de discussion dans le résumé scientifique. Cette étude constitue donc un point de départ vers des analyses plus systématiques et plus approfondies.

Quelques chiffres indiquant le nombre de résumés dans lesquels sont attestés certains phénomènes déterminés, sur un total de 105 résumés, viennent appuyer ces résultats :

Facteurs indiquant l'engagement :

1) présence du pronom nous dans 9 résumés ; présence deon dans 23 résumés ;

2) présence d'éléments nettement positifs dans 34 résumés et d'éléments nettement négatifs dans 18 résumés (il va de soi que ce sont là des calculs qui prêtent à discussion - ce qui est positif ou négatif est bien entendu quelque chose de très relatif) ;

Facteurs indiquant la discussion :

3) référence indiquée par un nom précis dans 17 résumés et par un nom avec date de parution de la recherche en question dans 4 résumés ;

4) présence de marqueurs polyphoniques : la negationne pas ou non est attestée dans 28 des 105 résumés et le connecteur mais dans 19 résumés.

Il faut admettre que les résultats obtenus n'ont qu'une valeur restreinte vu qu'il ne s'agit pas d'une étude quantitativement significative et que les constructions et expressions langagières observées ne sont pas examinées d'une manière suffisamment systématique et approfondie. Ces résultats justifient, cependant, l'utilité des ca- 
tégories proposées ainsi que du cadre théorique. Il faut également souligner que les analyses attestent de grandes variations de phénomènes : variations entre revues, entre disciplines et entre auteurs. Ce sont là des études à poursuivre.

Quoi qu'il en soit, les résultats des analyses permettent de conclure que le résumé scientifique est polyphonique. D'abord le locuteur est toujours présent, de manière plus ou moins explicite. Ensuite, il existe d'autres voix ou points de vue, manifestés soit par des références explicites à d'autres recherches, soit par des expressions langagières d'une manière implicite. Il est d'ailleurs difficile de s'imaginer des textes entièrement monophoniques ! Cependant, il faut souligner que la polyphonie peut être plus ou moins explicite. Les points de vue présentés ne peuvent pas toujours être attribués à des êtres discursifs précis. Voilà pourquoi la discussion, réalisée par des échanges de points de vue, peut apparaître comme peu claire et même absente dans le résumé scientifique. C'est là un point important par rapport aux observations faites par Berkenkotter et Huckin (1995:40), selon lesquelles la partie discussion de l'article scientifique semble être aussi importante que la partie introduction, dans la première lecture d'un tel article. Il faut, cependant, noter que, si la discussion n'est pas rendue d'une manière claire dans le résumé, c'est probablement dû, dans une certaine mesure à l'étendue du résumé. Il se peut également que la discussion soit trop complexe pour être rendue d'une manière satisfaisante dans le résumé.

De plus, il semble y avoir une tendance à attribuer une place importante aux résultats dans les résumés (comme l'indique aussi Lindeberg (à paraître) dans son étude sur des résumés tirés des domaines de la finance, du management et du marketing). Cependant, à part la préoccupation de signaler " the news value " de la contribution en question (voir Berkenkotter \& Huckin 1995), on pourrait se demander si l'argumentation et la polémique ne constituent pas des facteurs intéressants justement pour capter l'attention du lectorat. Il serait peut-être judicieux pour les résumés scientifiques - au moins pour les résumés du type informatif - de mettre encore plus en valeur la discussion menée dans l'article - le texte source du résumé.

Quelle que soit la raison de leur présence, des éléments langagiers manifestant engagement et discussion existent bel et bien dans les résumés scientifiques. C'est le cadre polyphonique qui nous a permis d'identifier ces éléments signalant des échanges de points de vue. Il faudrait toutefois vérifier ces résultats dans des recherches ultérieures se basant sur des matériaux plus étendus et où l'on tient compte des différences éventuelles entre cultures, disciplines, revues et chercheurs individuels. 


\section{Notes}

1. Le terme " auteur " ou " locuteur " renvoie à celui qui a rédigé le résumé, qui peut être ou non le rédacteur du texte source. Parce que les résumés étudiés dans notre corpus apparaissent concurremment avec leur texte original dans des revues, ils seront, vraisemblablement, des résumés d'auteur.

\section{Références}

Adam, J.-M. (1992). Les textes : types et prototypes Paris : Nathan.

Anscombre, J.-C. \& Ducrot, O. (1983). L'argumentation dans la langue. Liège : Mardaga.

Bakhtine, M. (1970). Problèmes de la poétique de Dostoïevski. Traduit par Guy Verret. Lausanne :Éd. L'Âge de l'Homme.

Bazerman, C. (1988). Shaping written knowledge: The genre and activity of the experimental article in science. Madison, Wis. : University of Wisconsin Press.

Berkenkotter, C. \& Huckin, T.N. (1995). Genre knowledge in disciplinary communication : Cognition/Culture/Power Hillsdale, N.J. : Lawrence Erlbaum.

Breivega, K. R. (2000). Den vitskaplege artikkelen som forskningsobjekt. Vitskapsteoretisk forankring og tekstkonstituering : Samanheng eller samanbrot? In T. Kinn \& R. B. Brodersen (Eds.), Språkvitskap og vitskapsteori. 10 nye innlegg til Dr.art.graden. Bergen : Ariadne.

Breivega, K. R. (à paraître). Epistemic modality as part of superstructural organization in academic prose. Paper presented at the 12th European Symposium on Language for Special Purposes, Bressanone/Brixen.

Dahl, T. (2000a). Text summarisation : From human activity to computer program. The problem of tacit knowledge. Hermes, 25, Danemark : Aarhus.

Dahl. T. (20oob). LEXICAL COHESION-BASED TEXT CONDENSATION. An evaluation of automatically produced summaries of research articles by comparison with author-written abstracts. Dissertation, University of Bergen/ Norwegian School of Economics and Business Administration.

Ducrot, O. (1984). Le dire et le dit. Paris : Minuit.

Fløttum, K. (1990). La nature du résumé scolaire. Analyse formelle et informative. Paris/Oslo : Didier Erudition/Solum (thèse). 
Fløttum, K. (1992). Polyphonic structure. In A.-C. Lindeberg et al. (Eds.), Nordic Research on Text and Discourse (pp.161-172). Abo Academy Press.

Fløttum, K. (1996). Written reformulation in a modular approach. International Journal of Applied Linguistics, 6(1), 65-79.

Fløttum, K. (1997). Cohérence textuelle et polyphonie. In B. Caron (Ed.) Proceedings of the 16th International Congress of Linguists (Paris 1997). Oxford : Pergamon/Elsevier, Sciences, CD-ROM, Paper no.o33o.

Fløttum, K. (1998). Le Mot du P.D.G. - descriptif ou polémique. In Y. Gambier (ed.), Discours professionnels en français. Berne : P. Lang,105-122.

Fløttum, K. (1999a). Typologie textuelle et polyphonie : quelques questions. Tribune 9, Université de Bergen, Institut d'Études romanes, 81-96.

Fløttum, K. (1999b). Linguistic polyphony : An introduction and some applications. In O. Dysthe (Ed.), The dialogical perspective and Bakhtin. University of Bergen : PLF Report2/99.

Fløttum, K. (à paraître a). De la phrase au texte : un pas en arrière ou une perspective prometteuse pour la linguistique textuelle? Actes du XXIIe Congrès international de Linguistique et Philologie romanes 1998, Bruxelles.

Fløttum, K. (à paraître b). La dimension énonciative dans les typologies textuelles. Actes du XIVe Congrès des Romanistes Scandinaves 1999, Université de Stockholm.

Frandsen, F. (1992). News discourse : The paratextual structure of news texts. In A.C. Lindeberg et al. (Eds.), Nordic research on text and discourse (pp. 147-157). Åbo Academy Press.

ISO. (1976). Documentation - Analyse pour les publications et la documentation. $\mathrm{1}^{\mathrm{re}}$ édition. ISO 214-1976(F). Genève : Organisation internationale de normalisation.

Laurendeau, P. (1990). Vers une typologie des tendenciels discursifs. Protée, printemps 1990, 125-133.

Liddy, E.D. (1991). The discourse-level structure of empirical abstracts : An exploratory study. Information Processing \& Management, 27(1), 55 - 81. 
Lindeberg, A.-C. (à paraître). Abstracting across disciplines : Rhetorical structures in research article abstracts in finance, management and marketing. ESP Journal.

Loffler-Laurian, A.-M. (1980). L'expression du locuteur dans les discours scientifiques. Revue de linguistique romane, 44, 135-157.

Norén, C. (1999). Reformulation et conversation. De la sémantique du topos aux fonctions interactionnelles. Uppsala : Université d'Uppsala.

Nølke, H. (1994). Linguistique modulaire : de la forme au sens Louvain : Peeters.

Nølke, H. (1999). La polyphonie : analyses littéraire et linguistique.Tribune, 9, 5-19. Université de Bergen, Institut d'études romanes.

Swales, J.M. (1990). Genre analysis : English in academic and research settings Cambridge : Cambridge University Press.

Tibbo, H.R. (1992). Abstracting across the disciplines : A content analysis of abstracts from the natural sciences, the social sciences, and the humanities with implications for abstracting standards and online information retrieval. Library and Information Science Research, 14(1), 31 - 56. Tribune, 9(1999). Université de Bergen, Institut d'études romanes.

Vassileva, I. (1998). Who am I/who are we in academic writing? A contrastive analysis of authorial presence in English, German, French, Russian and Bulgarian. International Journal of Applied Linguistics, 8(2), 163-190. Oslo : Novus Press. 